\title{
Star Cluster Systems in Interacting and Starburst Galaxies: A Multicolour Approach
}

\author{
Richard de Grijs \\ Institute of Astronomy, University of Cambridge, Madingley Road, Cambridge CB3 \\ OHA, UK
}

\begin{abstract}
The large majority of extragalactic star cluster studies done to date have essentially used two or three-passband aperture photometry, combined with theoretical stellar population synthesis models, to obtain age estimates. The accuracy to which this can be done depends on the number of observations through different (broad-band) filters available as well as, crucially, on the actual wavelength range covered. I show, based on the examples of the nearby starburst galaxies NGC 3310 and M82, that the addition of, in particular, near-infrared passbands to a set of optical filters greatly enhances our ability to disentangle age, metallicity and extinction parameters for star clusters with ages younger than a few Gyr. In addition, for the intermediate-age star cluster system in the fossil starburst region of M82 we find (i) a well-defined burst of cluster formation at slightly older ages than derived from previous estimates based on optical fluxes alone, and (ii) that by considering only the clusters originating in this burst of cluster formation, we uncover the first conclusive observational evidence for an unambiguous turn-over in the luminosity function of a coeval star cluster system with an age as young as $\sim 1$ Gyr.
\end{abstract}

\section{The cluster luminosity function as diagnostic}

The distribution of cluster brightnesses, known as the Cluster Luminosity Function (CLF), is one of the most important diagnostics for the study of extragalactic compact star cluster populations. For the old globular cluster (GC) systems the CLF shape is well-established: it is roughly Gaussian, with the peak or turn-over magnitude at $M_{V}^{0} \simeq-7.4$ and a FWHM of $\sim 3$ mag (Harris 1991, Whitmore et al. 1995, Harris et al. 1998). The well-studied young star cluster (YSC) population in the Large Magellanic Cloud, on the other hand, displays a power-law CLF (Elson \& Fall 1985, Elmegreen \& Efremov 1997).

Hubble Space Telescope (HST) observations have provided CLFs for young compact cluster systems in more distant galaxies and are continuing to do so. Although incompleteness effects often preclude detection of a turn-over in the CLF (e.g., Whitmore \& Schweizer 1995, Schweizer et al. 1996, Miller et al. 1997), in galaxies with YSC systems for which deep observations are available there is little evidence for an intrinsic turn-over (but see de Grijs, Bastian \& Lamers 2002b). In most of these cases, the CLF shapes are consistent with power laws down to the completeness threshold (but see Miller et al. 1997, de Grijs et al. 2001, 2002b).

GC formation models suggest that the distribution of the initial cluster masses (and, therefore, of the initial cluster luminosities) is closely approxi- 
mated by a power law (e.g., Harris \& Pudritz 1994, McLaughlin \& Pudritz 1996, Elmegreen \& Efremov 1997). The processes responsible for the depletion of, preferentially, low-luminosity, low-mass star clusters over time-scales of a Hubble time, leading to the Gaussian CLFs observed, include tidal interactions with the gravitational field of the parent galaxy and evaporation of stars through two-body relaxation within clusters. From the models of Gnedin \& Ostriker (1997) and Elmegreen \& Efremov (1997) it follows that any initial mass (or luminosity) distribution will shortly be transformed into peaked distributions. However, Vesperini $(2000,2001)$ has demonstrated that, due to dynamical friction effects affecting the high-mass clusters, considerable fine tuning of the model parameters is required to produce from an initial power-law distribution a Gaussian-type mass function with parameters similar to those observed for well-studied GC systems.

However, all of these models are valid only for time-independent Milky Waytype gravitational potentials; galaxy-galaxy interactions will obviously have a major effect on the resulting (time varying) gravitational potential, in which the dynamical star cluster evolution is likely significantly different.

\section{The importance of multicolour photometry}

Age spread effects in cluster systems in which cluster formation is still ongoing affect the observed CLF (Meurer 1995, Fritze-v. Alvensleben 1999, de Grijs et al. 2001, 2002a,b), which might in fact make an intrinsically Gaussian CLF appear as a power-law CLF (see, e.g., Miller et al. 1997). For young and intermediateage cluster systems it is very important to age date the individual clusters and to correct the observational CLF to a common age before interpreting their CLF.

Metallicities of YSCs produced in galaxy interactions, mergers or starbursts are an important discriminator against GCs formed in the early Universe. They are expected to correspond to the interstellar medium abundances of the interacting/starburst galaxies and are therefore most likely significantly higher than those of halo GCs in the Milky Way and other galaxies with old GC systems. Precise metallicity determinations for individual YSCs are therefore important to determine ages, and age spreads, from integrated colours.

Dust extinction is often very important in YSC systems. In particular the youngest post-burst galaxies and galaxies with ongoing starbursts often show strong and patchy dust structures and morphologies. Extinction estimates towards individual YSCs are therefore as important as individual metallicity estimates in order to obtain reliable ages and to be able to derive an age-normalised CLF or YSC mass function.

As part of an ongoing ASTROVIRTEL] project, we are in the process of assessing the systematic uncertainties in age, extinction and metallicity determinations for YSC systems inherent to the use of broad-band, integrated colours. We

\footnotetext{
1 The ASTROVIRTEL project is aimed at enhancing the scientific return of the STECF/ESO Archive. It offers the possibility to European users to exploit it as a virtual telescope with the assistance of the Archive management.
} 
have developed an evolutionary synthesis optimisation technique that can be applied to photometric measurements in a given number $N(N \geq 4)$ of broad-band passbands (see Anders, Fritze-v. Alvensleben \& de Grijs, these proceedings). The optimisation routine then simultaneously determines the best combination of age, extinction and metallicity from a comparison with the most up-to-date Göttingen simple stellar population (SSP) models (Schulz et al. 2002), to which we have added the contributions of an exhaustive set of gaseous emission lines and gaseous continuum emission.

The most important scientific issue we want to address is whether, and to what accuracy, we can determine the violent star formation histories of galaxies based on star cluster colour distributions and deep luminosity functions in several optical and/or near-infrared (NIR) passbands. Closely related to this issue is the question as to whether the star clusters formed in interacting galaxies will eventually evolve into the old GC systems we see today. The large majority of extragalactic star cluster studies done to date have essentially used two or three-passband aperture photometry, combined with theoretical stellar population synthesis models, to obtain age estimates (while in most cases metallicities were fixed at solar values). The accuracy to which this can be done obviously depends on the number of observations through different (broad-band) filters available as well as, crucially, on the actual wavelength range covered.

\section{A case for additional near-infrared observations}

As a pilot case for our study of systematic effects introduced by the choice of any particular passband combination we decided to concentrate on NGC 3310. This choice was made because of (i) the large and homogenous set of observations of this galaxy available in the HST archive, and (ii) the large number of prior extragalactic star cluster studies available in the literature to which we can compare our results.

NGC 3310 is a local $(D=12.5 \mathrm{Mpc})$, very active starburst galaxy with high global star formation efficiency. A number of morphological and kinematical peculiarities suggest that NGC 3310 was affected by a major gravitational disturbance, which led to high, possibly sustained, star formation rates in the past $~ 100$ Myr (cf. Balick \& Heckman 1981). The bar-driven star formation scenario suggested by Conselice et al. (2000), combined with the recent infall of a companion galaxy, is the currently most attractive scenario for this: it provides a natural explanation for the low metallicity observed in the star-forming knots near the centre, while it also explains why we observe concentrated star formation in star clusters or very luminous HiI regions in a tightly-wound ringlike structure surrounding the centre. All of the observational evidence points at very recent star formation in the star clusters and HII regions, and a time since the interaction of $\leq 10^{7-8}$ yr (e.g., Balick \& Heckman 1981, Smith et al. 1996).

In the galaxy's central $22^{\prime \prime} .55 \times 22^{\prime \prime} 65$ region covered by all of the WFPC2 and NICMOS observations available in the HST archive, we detected 289 pointlike (or marginally extended) star cluster candidates brighter than $4 \times$ the r.m.s. 

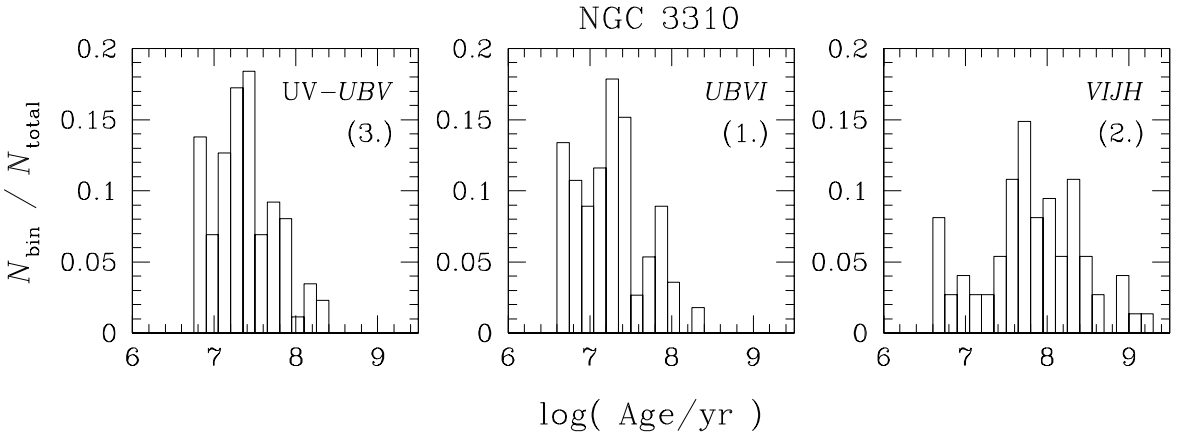

Fig. 1. Normalised age distributions of the NGC 3310 clusters based on three choices of broad-band passband combinations, as described in the text.

noise of the background in both of the F606W and F814W source lists. To assess the effects of choosing a particular passband combination on the final results, we applied our fitting technique to our flux measurements in four sets of passbands (see Fig. 1):

1. a subset of only optical passbands: F336W, F439W, F606W and F814W;

2. a red-selected passband combination: F606W, F814W, F110W and F160W;

3. a blue-selected passband combination: F300W, F336W, F439W and F606W;

4. the full set of seven passbands, from F300W to F160W.

It is immediately clear from Fig. 1 that the resulting age distribution of an extragalactic star cluster system based on integrated broad-band colours is a sensitive function of the passbands covered by the observations. The cause for these significantly different age distributions with and without the inclusion of the near-UV and/or NIR filters is the degeneracy between age and extinction, and to a lesser extent also between age and metallicity, for the optical (and UV) colour combinations. In particular for the younger ages, the least-squares fitting routines tend to overestimate the extinction without NIR information, and therefore underestimate the ages. As I will show below (see also de Grijs et al. 2002a; Parmentier, de Grijs \& Gilmore, these proceedings) for the star cluster population in M82, the addition of NIR passbands greatly enhances our ability to disentangle age, metallicity and extinction parameters for YSCs with ages younger than a few Gyr. Although the full interpretation and details of our study of systematic uncertainties will be described in detail in a forthcoming paper, here I want to point out that while the actual age estimates are a sensitive function of passband choice, the relative age distributions resulting from all of the different passband combinations indicate a young age peak (age $\leq 10^{7} \mathrm{yr}$ ) and a major burst of cluster formation between $10^{7}$ and $10^{8}$ yr ago. These age estimates are fully consistent with previously published estimates, sometimes based on significantly more sophisticated techniques. In fact, while the clusters in the older burst are smoothly distributed throughout the centre of the galaxy 

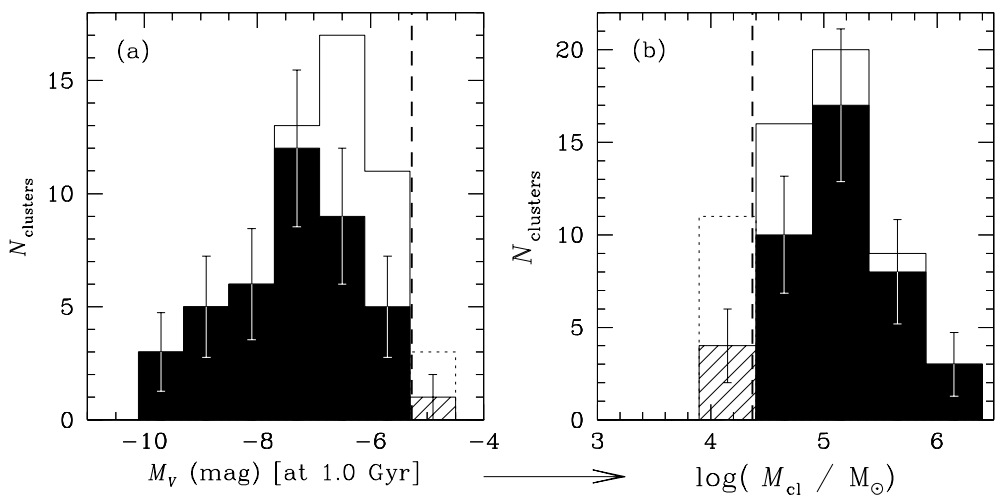

Fig. 2. Age-normalised CLF and corresponding mass distribution of the M82 clusters formed in the burst of cluster formation, $8.4 \leq \log$ (Age/yr) $\leq 9.4$. The shaded histograms correspond to the clusters with well-determined ages; the open histograms represent the entire cluster sample in the age range of the burst. The vertical dashed line is our selection limit.

and its nuclear ring, the youngest star clusters are predominantly located in the (very young) "Jumbo" region and the actively star forming northern spiral arm.

\section{The M82 fossil starburst revisited}

In a recent study focusing on the fossil starburst site in the nearby, "prototypical" starburst galaxy M82 we found a large population of $(\sim 110)$ evolved compact star clusters (de Grijs et al. 2001), whose properties appear to be consistent with them being evolved (and therefore faded) counterparts of the young star clusters detected in the galaxy's active core (O'Connell et al. 1995). Based on broad-band optical colours only and on comparison with stellar evolutionary synthesis models, we estimated ages for these clusters from $\sim 30$ Myr to over 10 Gyr, with a peak near 650 Myr. We have since obtained new age and mass estimates for these star clusters, based on improved fitting methods to the full spectral energy distribution, including the F110W and F160W fluxes (de Grijs et al. 2002a). Our new age estimates confirm the peak in the age histogram attributed to the last tidal encounter with M81, but at slightly older ages than previously published, $\log \left(t_{\mathrm{peak}} / \mathrm{yr}\right)=9.04$. This, again, underscores the importance of including NIR passbands when trying to determine global cluster properties from integrated photometry.

As shown in Fig. 2, both the mass distribution and the CLF (corrected to a common age of $1.0 \mathrm{Gyr}$ ) of the clusters formed during the burst, defined as $8.4<\log ($ Age $/$ yr $)<9.4$, show an unambiguous turn-over at an order of magnitude more massive, and about 2 magnitudes brighter, than our detection limit, respectively (de Grijs et al. 2002b). This turn-over is not due to selection 
Richard de Grijs

effects, which we do, in fact, understand very well for the clusters formed in the burst (see de Grijs et al. 2002a). This is the first time that a clear turnover has been detected unambiguously for a coeval star cluster system with an intermediate age as young as $\sim 1$ Gyr. We have also shown that with the very short characteristic cluster disruption time-scale governing M82's fossil starburst region (de Grijs et al. 2002a), its cluster mass distribution will evolve towards a higher characteristic mass scale than for the Galactic GCs by the time it reaches a similar age (de Grijs et al. 2002b). We argue, therefore, that this evidence, combined with the similar cluster sizes (de Grijs et al. 2001), lends strong support to a scenario in which the current slightly evolved M82 cluster population will eventually evolve into a significantly depleted old Milky Way-type GC system dominated by a small number of high-mass clusters. This implies that progenitors of Milky Way-type GCs, which were once thought to be the oldest building blocks of galaxies, are still forming today in galaxy interactions and mergers. However, they will likely be more metal-rich than the present-day old GC systems.

\section{Acknowledgements}

I acknowledge support from ASTROVIRTEL, a project funded by the European Commission under FP5 Contract No. HPRI-CT-1999-00081. Funding for this work was provided by the Particle Physics and Astronomy Research Council (PPARC) in the UK.

\section{References}

1. Balick B., Heckman T., 1981, A\&A, 96, 271

2. Conselice C.J., Gallagher J.S., Calzetti D., Homeier N., Kinney A.L., 2000, AJ, 119, 79

3. de Grijs R., Bastian N., Lamers H.J.G.L.M., 2002a, MNRAS, submitted

4. de Grijs R., Bastian N., Lamers H.J.G.L.M., 2002b, ApJL, submitted

5. de Grijs R., O'Connell R.W., Gallagher III J.S., 2001, AJ, 121, 768

6. Elmegreen B.G., Efremov Y.N., 1997, ApJ, 480, 235

7. Elson R.A.W., Fall S.M., 1985, PASP, 97, 692

8. Fritze-v. Alvensleben U., 1999, A\&A, 342, L25

9. Gnedin O.Y., Ostriker J.P., 1997, ApJ, 474, 223

10. Harris W.E., 1991, ARA\&A, 29, 543

11. Harris W.E., Harris G.L.H., McLaughlin, D.E., 1998, AJ, 115, 1801

12. Harris W.E., Pudritz R.E., 1994, ApJ, 429, 177

13. McLaughlin D.E., Pudritz R.E., 1996, ApJ, 457, 578

14. Meurer G.R., 1995, Nat, 375, 742

15. Miller B.W., Whitmore B.C., Schweizer F., Fall S.M., 1997, AJ, 114, 2381

16. O'Connell R.W., Gallagher J.S., Hunter D.A., Colley W.N., 1995, ApJ, 446, L1

17. Schweizer F., Miller B.W., Whitmore B.C., Fall S.M., 1996, AJ, 112, 1839

18. Smith D.A., et al., 1996, ApJ, 473, L21

19. Vesperini E., 2001, MNRAS, 318, 841

20. Vesperini E., 2001, MNRAS, 322, 247

21. Whitmore B.C., Schweizer F., 1995, AJ, 109, 960

22. Whitmore B.C., Sparks W.B., Lucas R.A., Macchetto F.D., Biretta J.A. 1995, ApJ, 454, L73 